\title{
Proteomics-based investigation of multiple stages of OSCC development indicates that the inhibition of Trx-1 delays oral malignant transformation
}

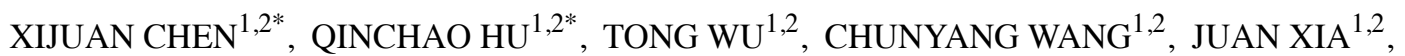 \\ LINGLAN YANG ${ }^{1,2}$, BIN CHENG ${ }^{1,2}$ and XIAOBING CHEN ${ }^{1,2}$ \\ ${ }^{1}$ Guanghua School of Stomatology, Hospital of Stomatology, Sun Yat-sen University; \\ ${ }^{2}$ Guangdong Provincial Key Laboratory of Stomatology, Guangzhou, Guangdong 510060, P.R. China
}

Received September 21, 2017; Accepted December 31, 2017

DOI: 10.3892/ijo.2018.4235

\begin{abstract}
The majority of cases of oral squamous cell carcinoma (OSCC) develop from oral potentially malignant disorders, which have been confirmed to be involved in chronic oxidative stimulation. However, no effective treatment approaches have been used to prevent the development of dysplasia into cancerous lesions thus far. In the present study, a well-established OSCC model was used to detect proteomics profiles at different stages during oral malignant transformation. Of the 15 proteins that were found to be upregulated in both the dysplasia and carcinoma stages, the oxidative stressassociated proteins, thioredoxin-1 (Trx-1), glutaredoxin-1 and peroxiredoxin- 2 were note as the proteins with significant changes in expression Trx-1 was identified to be the most significantly upregulated protein in the precancerous stage. Validation experiments confirmed that Trx-1 was overexpressed both in dysplasia and cancerous tissue samples, and the inhibition of Trx-1 was able to promote the apoptosis of OSCC cells under hypoxic conditions. Furthermore, the experimental application of a Trx-1-specific inhibitory agent in an animal model led to a lower cancerization rate and a delay in tumor formation. The possible mechanisms were associated with the increased apoptosis via a reactive oxygen species (ROS)dependent pathway. Taken together, our findings indicate that Trx-1 may be an important target for delaying oral malignant transformation, which provides a novel therapeutic strategy for the prevention and treatment of OSCC.
\end{abstract}

Correspondence to: Dr Xiaobing Chen or Professor Bin Cheng, Guanghua School of Stomatology, Hospital of Stomatology, Guangdong Provincial Key Laboratory of Stomatology, Sun Yat-sen University, 56 Lingyuanxi Road, Guangzhou, Guangdong 510060, P.R. China

E-mail: chenxb7@mail.sysu.edu.cn

E-mail: chengbin@mail.sysu.edu.cn

${ }^{*}$ Contributed equally

Key words: oral potentially malignant disorders, oral squamous cell carcinoma, proteomics, thioredoxin 1, oxidative stress, malignant transformation

\section{Introduction}

As a type of solid tumor, oral squamous cell carcinoma (OSCC) accounts for $\sim 90 \%$ of oral malignancies, and remains associated with a poor prognosis and low survival rate, despite advances in diagnosis and therapy (1,2). Approximately $20 \%$ of OSCC cases develop from epithelial dysplasia lesions, so-called oral potentially malignant disorders (OPMDs), such as oral leukoplakia and erythroplakia (3). Epidemiological surveys have confirmed that OPMDs and OSCC are often influenced by continuous high-risk behaviors, such as the use of tobacco, alcohol consumption and betel nut use, which can generate chronic oxidative stress $(4,5)$. The effective early prevention and detection of OPMDs may play a pivotal role in decreasing the incidence of OSCC. However, no effective treatment approaches have been used to prevent the development of dysplasia into cancerous lesions thus far. Consequently, there is a critical need for the discovery of novel therapeutic targets for OPMDs for the early prevention of OSCC (6).

Proteomics analysis has become increasingly powerful for the elucidation of protein expression and cell signaling pathways (7). Numerous studies have described the preliminary application of proteomics in the identification of biomarkers for OSCC (8-10), while few have used it to identify the development of OPMDs (11). This may due to the fact that it is difficult to trace the progress of oral malignant transformation in individual patients, since this is often a lengthy process. A well-established OSCC model, which can mimic naturallyoccurring OSCC within an observable duration, is induced by the tumorigenic compound, 4-nitroquinoline 1-oxide (4NQO). It is one of the most extensively studied animal systems due to its close similarity to human oral malignant transformation at the histological and molecular levels (12). This animal model has been widely used in the study of OPMDs (13). Furthermore, Lan et al (14) demonstrated that 4NQO induced oxidative DNA damage and activated the nuclear factor (erythroidderived 2)-like 2 (Nrf2) pathway in the mouse tongue.

In the present study, the profile of differentially-expressed proteins during $4 \mathrm{NQO}$-induced oral carcinogenesis was investigated by iTRAQ-based proteomics followed by quantitative verification. The oxidative stress-associated proteins, 
such as thioredoxin-1 (Trx-1), glutaredoxin-1 (Grx-1) and peroxiredoxin-2 (Prx-2), were noted as the proteins with the most significant changes in expression. Among these proteins, Trx-1, which is a member of the thioredoxin system and plays an important role in maintaining redox balance, seemed to be the most significantly upregulated protein in the precancerous stage. A delay in tumor formation and a lower cancerization rate was observed following the inhibition of Trx-1 with an irreversible inhibitor, 1-methylpropyl 2-imidazolyl disulfide (PX-12) (15), in the animal model, which indicated that Trx-1 may be a promising intervention target for OSCC development.

\section{Materials and methods}

Established of rat model of 4NQO-induced oral carcinogenesis. The rat model of 4NQO-induced oral carcinogenesis was established as described previously (16). A total of 20 male Sprague-Dawley (SD) rats (4 weeks of age and weighing 75-100 g) were divided into 2 groups. In the experimental group $(n=14)$, rats were fed daily with 20 ppm 4NQO (Sigma-Aldrich; Merck KGaA, Darmstadt, Germany) solution in their drinking water. At weeks 16-24, the experimental rats were sacrificed, as visible lesions of tongue dysplasia or squamous cell carcinoma had developed. The normal control group $(n=6)$ was fed with distilled water only. The rat tongues were harvested and sagittally cut into 2 halves. One half was fixed in $10 \%$ buffered formalin, embedded in paraffin and cut into 4-mm-thick sections for hematoxylin and eosin staining to confirm the pathological diagnosis. The other half was collected and stored in liquid nitrogen. Finally, 6 dysplasia samples (1 mild, 3 moderate and 2 severe) and 8 well-differentiated carcinoma samples were collected. All the animal procedures were conducted in accordance with the Guidelines for the Care and Use of Laboratory Animals and were approved by the Institutional Animal Care and Use Committee at Sun Yat-sen University (Guangzhou, China).

$P X-12$ intervention in the rat model of $4 N Q O$-induced oral carcinogenesis. Another 22 male SD rats (4 weeks of age and $75-100 \mathrm{~g}$ in weight) were divided into 2 groups. In the experimental group $(n=16)$, the rats were fed daily with 20 ppm 4NQO (Sigma-Aldrich; Merck KGaA) solution in their drinking water for 24 weeks. The rats in the normal control group $(n=6)$ were fed with distilled water only. At week 18, the experimental rats were divided into 2 groups as visible lesions of tongue dysplasia had appeared. One group was injected with PX-12 (12 mg/kg) weekly via the tail vein. The other group and the normal control group were injected with an equal amount of saline. The rats were sacrificed at week 24. The tongues were dissected, and a longitudinal midlingual incision was made. The specimens were collected and analyzed as described below.

Proteomics analysis. The normal control, dysplasia and squamous cell carcinoma specimens $(n=6,6$ and 8 , respectively) were digested in $1.8 \mathrm{U} / \mathrm{ml}$ dispase II (Roche Applied Science, Penzberg, Germany) at $4^{\circ} \mathrm{C}$ overnight. The epithelial tissues were enzymatically and mechanically separated from the connective tissues. The samples were detected using
iTRAQ-based proteomic analysis at the Beijing Genomics Institute (BGI; Shenzhen, China). The protocol for proteomics analysis was conducted according to the BGI guidelines, as previously described (7). In this experiment, the total proteins of the epithelial tissues from the 3 groups were extracted and digested into peptides, respectively. The desalted peptides were labeled with iTRAQ reagents (SCIEX, Framingham, MA, USA). The normal control, dysplasia and squamous cell carcinoma were labeled with 114, 116 and 119 iTRAQ tags, respectively [normal (N)-114, dysplasia (D)-116 and carcinoma (C)-119]. The LTQ-Orbitrap-Velos hybrid mass spectrometer (Thermo Fisher Scientific, Inc., Waltham, MA, USA) coupled with strong cation exchange chromatography (SCX) and liquid chromatography (Thermo Fisher Scientific, Inc.) was used to analyze the mixed peptides.

Database search and quantitative proteomics analysis. All raw data files were searched using Mascot 2.3.02 (Matrix Science Ltd., London, UK) against the Rattus RefSeq database (www.ncbi.nlm.nih.gov/protein) and the Uniprot Human database (www.uniprot.org), containing 29,964 sequences. The following identification parameters were selected: MS/MS ion search; enzyme, trypsin; fragment mass tolerance, $\pm 0.02 \mathrm{Da}$; mass values, monoisotopic mass; max missed cleavages, 1; peptide mass tolerance, $10 \mathrm{ppm}$; variable modifications, Gln-> pyro-Glu (N-termQ), oxidation (M) and iTRAQ8plex (Y); fixed modifications, carbamidomethyl (C), iTRAQ8plex (N-term) and iTRAQ8plex (K). The identified proteins and peptides were filtered with confidence corresponding to the 1\% FDR. The ratios of 116:114 and 119:114 were calculated. A fold change of $\geq 1.5$ or $\leq 0.67$ and a $P$-value $<0.05$ were set as the thresholds for screening differentially expressed proteins.

Patients and samples. Specimens were obtained from the oral mucosa of 60 subjects, including patients with OSCC $(n=35)$, patients with oral leukoplakia $(n=15)$ and healthy control individuals $(n=10)$. The human healthy mucosa tissues were obtained from the cheeks and gingiva of patients who underwent orthognathic surgery. The diagnosis was based on clinical appearance and histological analysis. Each subject provided written consent to participate after being informed about the aims and protocol of the research. This study was approved by the Ethics Committee of Guanghua School of Somatology, Sun Yat-sen University.

Cell culture and induction of hypoxia. The CAL33 cell line was originally purchased from the Leibniz Institute DSMZ-German Collection of Microorganisms and Cell Cultures GmbH (Braunschweig, Germany). The HSC6 cell line was obtained from the National Cancer Center Research Institute (Tokyo, Japan). Both cell lines were preserved at the Guangdong Provincial Key Laboratory of Stomatology. The cells were cultured in Dulbecco's modified Eagle's medium (DMEM) supplemented with $10 \%$ fetal bovine serum (FBS) (both from Gibco; Thermo Fisher Scientific, Inc.) and 1\% penicillin-streptomycin (Gibco, Grand Island, NY, USA) at $37^{\circ} \mathrm{C}$ in a $20 \% \mathrm{O}_{2}$ and $5 \% \mathrm{CO}_{2}$ humidified incubator (normoxic conditions). Hypoxic conditions were induced in a tri-gas incubator at $37^{\circ} \mathrm{C}$ with $1 \% \mathrm{O}_{2}, 5 \% \mathrm{CO}_{2}$ and $94 \% \mathrm{~N}_{2}$. The cells were transferred into the specific incubator where 
necessary. PX-12 was dissolved in dimethyl sulfoxide (DMSO) at $100 \mathrm{mM}$ as a stock solution. N-acetyl cysteine (NAC) (all from Sigma-Aldrich; Merck KGaA) was diluted to $500 \mathrm{mM}$ by filtering. In this study, the cells were treated with $5 \mathrm{mM} \mathrm{NAC}$ for $1 \mathrm{~h}$ prior to treatment with PX-12, and $0.03 \%$ DMSO was used as a vehicle control.

Cell proliferation assay. Cell viability was detected by Cell Counting Kit-8 (CCK-8) assay (Dojindo, Kumamoto, Japan). The HSC6 $\left(5 \times 10^{3}\right.$ cells/well) and CAL33 cells $\left(6 \times 10^{3}\right.$ cells/well $)$ were seeded into 96-well plates. After $24 \mathrm{~h}$, the cells were treated with various concentrations of PX-12 $(0-50 \mu \mathrm{M})$ for $24 \mathrm{~h}$. Prior to the detection of the absorbance at $450 \mathrm{~nm}$ using a microplate reader (Thermo Fisher Scientific, Inc.) the cells were incubated with CCK- 8 reagent $(10 \mu \mathrm{l} /$ well $)$ for $2 \mathrm{~h}$ at $37^{\circ} \mathrm{C}$. All experiments were performed in triplicate.

Cell invasion assay. The invasion assays were performed using 24-well Transwell units (BD Biosciences, Franklin Lakes, NJ, USA). Matrigel (50 $\mu \mathrm{l})$ diluted with serum-free DMEM (1:5) was coated on the upper chamber. Subsequently, $8 \times 10^{4}$ cells in $200 \mu \mathrm{l}$ serum-free medium with or without PX-12 (0, 10, 20 or $40 \mu \mathrm{M})$ were seeded in the upper chamber of the system. In the experiments with NAC, the cells were treated with NAC for $1 \mathrm{~h}$ prior to the addition of PX-12. A total of $600 \mu$ DMEM with $10 \%$ FBS was added to the bottom wells. The cells were incubated for $24 \mathrm{~h}$. The invading cells on the bottom membrane of the upper chamber were fixed with $4 \%$ paraformaldehyde and stained with $0.4 \%$ crystal violet. Invading cell numbers were counted in 5 random fields (original magnification, x100).

Cellular apoptosis assay. For apoptosis assay, the Annexin V-FLUOS/propidium iodide (PI) double-staining apoptosis detection kit (Roche Diagnostics GmbH, Mannheim, Germany) was used. The OSCC cells were treated with various concentrations of PX-12 for $24 \mathrm{~h}$, following pretreatment with or without $5 \mathrm{mM}$ NAC for $1 \mathrm{~h}$. The cells were collected and stained with $5 \mu \mathrm{l}$ Annexin V-fluorescein isothiocyanate and $5 \mu \mathrm{l}$ PI, according to the manufacturer's instructions. The acquisition and analysis of the apoptosis data were performed using a flow cytometer (FACSCalibur; BD Biosciences). Basal apoptosis was determined using the same method in control cells.

Terminal deoxynucleotidyltransferase-mediated dUTP nick end labelling (TUNEL) assay. TUNEL assays were used to identify the apoptotic cells using the FragEL ${ }^{\mathrm{TM}}$ DNA Fragmentation Detection kit (Calbiochem; EMD Chemicals Inc., Gibbstown, NJ, USA) according to the manufacturer's instructions. Briefly, the tissue sections were deparaffinized, rehydrated and incubated with proteinase $\mathrm{K}$ for $15 \mathrm{~min}$. Following treatment with $3 \% \mathrm{H}_{2} \mathrm{O}_{2}$ for $5 \mathrm{~min}$, the sections were incubated with terminal deoxynucleotidyl transferase (TdT) enzyme with TdT buffer and biotin-tagged nucleotides in a humidified chamber at $37^{\circ} \mathrm{C}$. Tagged nucleotides were detected using HRP solution. After washing, the sections were stained with diaminobenzidine (DAB) solution and counterstained with hematoxylin. For the evaluation of the slides, 100 tumor or epithelial cells were counted per high-power field (original magnification, $\mathrm{x} 400$ ).
Intracellular reactive oxygen species (ROS) detection. The intracellular ROS levels were measured using a dichlorofluorescein assay (Beyotime Institute of Biotechnology, Haimen, China). 2,7-Dichlorodihydrofluorescein diacetate (DCFH-DA) was used to evaluate the generation of ROS during oxidative damage. The cells were incubated with $100 \mu \mathrm{M}$ DCFH-DA for 20 min after being washed 3 times in serum-free medium. Finally, the cells were harvested and DCFH-DA fluorescence was assessed using a flow cytometer (FACSCalibur; BD Biosciences) at 494/525 nm. All experiments were performed in triplicate.

Immunohistochemistry (IHC). IHC staining was performed according to the manufacturer's instructions. Tissue sections were incubated with primary antibodies against Trx-1 (1:600, \#2429; Cell Signaling Technology, Inc., Danvers, MA, USA), hypoxia-inducible factor (HIF)-1 $\alpha(1: 500, a b 1)$ and Bax (1:100, ab32503) (both from Abcam, Cambridge, MA, USA) overnight at $4^{\circ} \mathrm{C}$, after being blocked in normal goat serum for $20 \mathrm{~min}$. Subsequently, the sections were incubated with peroxidaseconjugated goat anti-rabbit secondary antibody (GK600510; Gene Tech, Shanghai, China) for $30 \mathrm{~min}$ at room temperature. Finally, the slides were visualized with DAB (R\&D Systems, Inc., Minneapolis, MN, USA) for antigen detection and counterstained with hematoxylin. All steps were separated by phosphate-buffered saline (PBS) or PBST washes. The expression of proteins was quantified using a visual grading system based on the extent of staining (percentage of positive cells graded on a scale from $0-3$ as follows: $0,<5 \% ; 1,5-30 \%$; $2,30-70 \%$; and $3,>70 \%$ ) and the intensity of staining (graded on a scale from $0-3$ as follows: 0 , none; 1 , weak; 2 , moderate; and 3, strong) (17). A total of 5 representative fields at $x 400$ magnification were evaluated.

Western blot analysis. The cells were lysed with RIPA buffer supplemented with protease (both from Sigma-Aldrich; Merck $\mathrm{KGaA}$ ) and phosphatase inhibitors (Roche Applied Science). A BCA protein assay kit (ComWin Biotech Co., Ltd., Beijing, China) was used to measured the concentrations of the lysates. The lysates were then incubated at $99^{\circ} \mathrm{C}$ for $5 \mathrm{~min}$ and mixed with loading buffer (4:1; ComWin Biotech Co., Ltd.). The samples $(30 \mu \mathrm{g} /$ lane $)$ were separated on 10 or $12 \%$ SDS-PAGE gels and electrophoretically transferred onto a PVDF membrane (EMD Millipore, Billerica, MA, USA). The membrane was blocked in 5\% non-fat milk for $1 \mathrm{~h}$ at room temperature and then incubated with primary antibodies against Trx-1 (1:1,000, \#2429; Cell Signaling Technology, Inc.), HIF-1 $\alpha$ (1:1,000, ab1), Bax (1:1,000, ab32503) (both from Abcam), Bcl-2 $(1: 2,000, \# 2870 s), \beta$-actin $(1: 1,000, \# 4970 s)$, poly(ADP-ribose) polymerase (PARP) and cleaved PARP (1:1,000; Cle-PARP; \#9532s) (all from Cell Signaling Technology, Inc.) overnight at $4^{\circ} \mathrm{C}$, respectively. Subsequently, the membrane was washed in TBST 3 times and incubated with HRP-conjugated secondary antibody (1:3,000, \#7074s; Cell Signaling Technology, Inc.) for $1 \mathrm{~h}$ at room temperature. The immunoreactive bands were visualized with an enhanced chemiluminescence detection system (EMD Millipore). Immunoreactive bands were quantified by densitometry with ImageJ 1.48 (National Institutes of Health, Bethesda, MD, USA). Similar results were obtained from 3 independent experiments. 
A

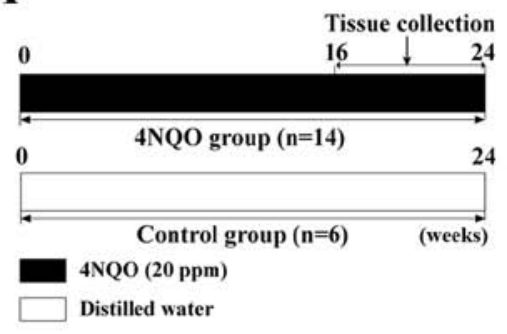

B

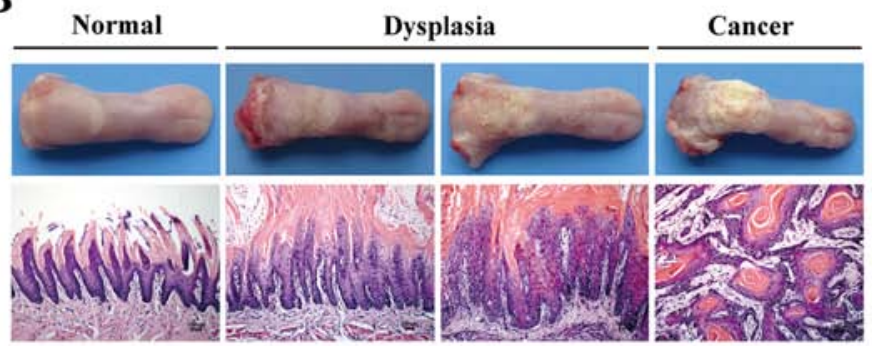

Human
C

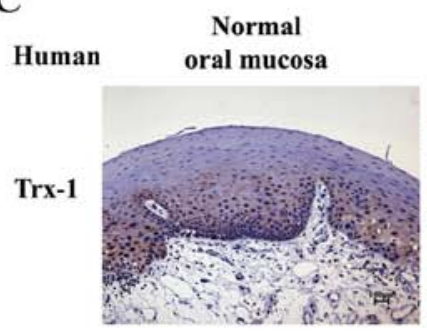

Oral leukoplakia

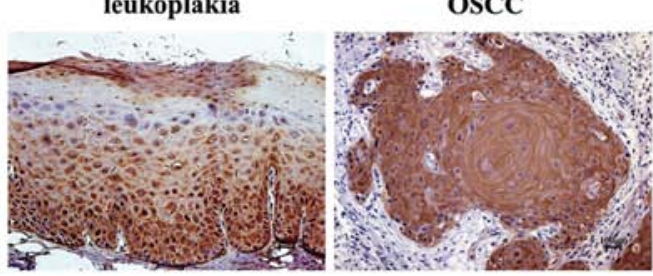

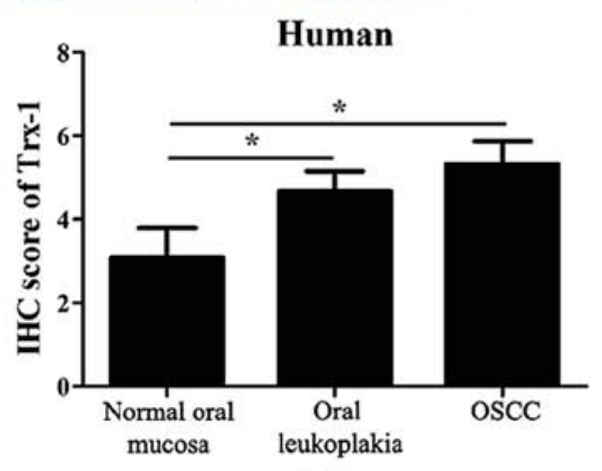

Rat

D

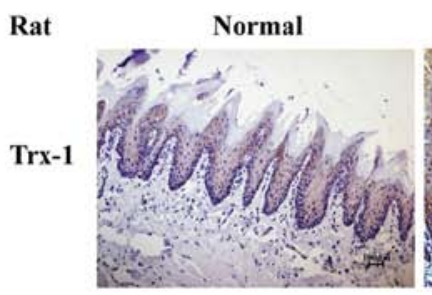

Dysplasia

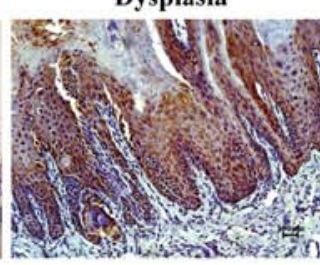

Cancer

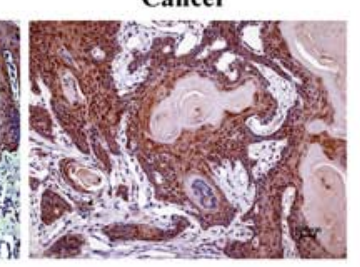

Figure 1. Establishment of the rat model of 4-nitroquinoline 1-oxide (4NQO)-induced oral carcinogenesis and the expression of thioredoxin-1 (Trx-1) during oral mucosal malignant transformation. (A) Schematic diagram of the establishment of the rat model of 4NQO-induced oral carcinogenesis. (B) Tissue specimens with hematoxylin and eosin staining (magnification, x200). Trx-1 expression was closely associated with oral mucosal malignant transformation. Representative images and graphs of Trx-1 immunohistochemistry (IHC) staining in normal, dysplasia and oral squamous cell carcinoma (OSCC) epithelia from (C) the human oral cavity and (D) the rat model of 4NQO-induced oral carcinogenesis. IHC scores are shown as the means \pm standard deviation (magnification, $\mathrm{x} 200) ;{ }^{*} \mathrm{P}<0.05$.

Statisticalanalysis. All results shown represent the means \pm standard deviation from triplicate experiments performed in a parallel manner, unless otherwise indicated. Statistical analyses were performed using a two-tailed Student's t-test, Mann-Whitney U test, Fisher's exact test and one-way ANOVA, where appropriate. A value of $\mathrm{P}<0.05$ was considered to indicate a statistically significant difference.

\section{Results}

Proteomics profiles of different stages of carcinogenesis in rats induced by $4 N Q O$. To explore protein profiles during oral carcinogenesis, a rat model of 4NQO-induced oral carcinogenesis was established (Fig. 1A and B). The epithelia from the normal, dysplasia and squamous cell carcinoma stages were collected for proteomics analysis. In this experiment, proteins were found to be differentially expressed in the specimens at the dysplasia stage (74 upregulated and 35 downregulated) and squamous cell carcinoma stage (119 upregulated and 170 downregulated) compared with the normal group specimens (data not shown). In total, 15 proteins were upregulated in both the dysplasia and carcinoma stages (Table I). Notably, the Trx, Grx and Prx proteins, which have been characterized as 'guardians' of the intracellular redox state (18), were indicated to be upregulated during oral carcinogenesis, including Trx-1, Grx-1 and Prx-2. Among these three oxidation-associated proteins, Trx-1 was identified to be the most upregulated protein among the 3 oxidation-associated proteins in the dysplasia stage (Table I).

Trx-1 is overexpressed during oral carcinogenesis. To confirm the expression pattern of Trx-1 during oral carcinogenesis, IHC was performed. The results demonstrated that Trx-1 expression gradually increased during oral malignant transformation, both in the human samples and the rat specimens. As shown in Fig. 1C, none or weak Trx-1 staining was detected in the human normal oral mucosa (the score of Trx-1 positive staining was $3.08 \pm 0.72$ ), while the staining of Trx-1 was particularly prominent in the abundant epithelial cytoplasm and nuclei in the oral leukoplakia $(4.68 \pm 0.47)$ and OSCC samples $(5.32 \pm 0.55)$. These scores were significantly higher compared with the normal samples $(\mathrm{P}<0.05)$. The 
Table I. Differentially expressed proteins in both the dysplasia and carcinoma stages.

\begin{tabular}{|c|c|c|c|c|c|}
\hline \multirow[b]{2}{*}{ Protein name } & \multicolumn{3}{|c|}{ Peptide detection } & \multicolumn{2}{|c|}{ Fold change } \\
\hline & Score & Coverage $(\%)$ & Unique peptide & D-116/N-114 & C-119/N-114 \\
\hline \multicolumn{6}{|l|}{ Upregulated proteins } \\
\hline Thioredoxin-1 & 105 & 31.4 & 4 & 1.869 & 1.503 \\
\hline Repetin & 1237 & 15.1 & 2 & 1.585 & 2.142 \\
\hline Plasminogen activator inhibitor 1 RNA-binding protein & 109 & 6.1 & 2 & 2.101 & 3.231 \\
\hline Peroxiredoxin-2 & 522 & 44.7 & 8 & 1.582 & 1.574 \\
\hline Nucleoside diphosphate kinase A & 184 & 45.4 & 3 & 1.580 & 2.136 \\
\hline Hepatoma-derived growth factor & 61 & 7.6 & 2 & 1.773 & 2.005 \\
\hline Glutaredoxin-1 & 169 & 16.8 & 2 & 1.658 & 1.555 \\
\hline Fatty acid-binding protein, epidermal & 496 & 22.2 & 3 & 1.558 & 1.942 \\
\hline Family with sequence similarity 83 , member $\mathrm{H}$ & 47 & 0.8 & 1 & 1.866 & 2.178 \\
\hline Eukaryotic translation elongation factor- $1 \beta 2$ & 259 & 16.4 & 3 & 2.101 & 1.713 \\
\hline Elongation factor $1-\delta$ & 224 & 4.8 & 3 & 1.629 & 2.042 \\
\hline Cystatin-A & 403 & 20.4 & 2 & 2.392 & 1.883 \\
\hline Clathrin light chain B & 131 & 10.5 & 3 & 2.028 & 2.055 \\
\hline Calmodulin-like protein 3 & 332 & 22.1 & 2 & 1.736 & 3.797 \\
\hline Barrier-to-autointegration factor & 206 & 40.4 & 2 & 1.527 & 1.505 \\
\hline \multicolumn{6}{|l|}{ Downregulated proteins } \\
\hline Phosphate carrier protein, mitochondrial & 75 & 6.2 & 2 & 0.635 & 0.362 \\
\hline Myotilin & 130 & 2.8 & 1 & 0.492 & 0.351 \\
\hline MICOS complex subunit & 229 & 6.3 & 1 & 0.653 & 0.420 \\
\hline Cytochrome c oxidase subunit $6 \mathrm{C}-2$ & 40 & 17.1 & 2 & 0.444 & 0.382 \\
\hline Cytochrome b-c 1 complex subunit 2 & 334 & 14.8 & 5 & 0.646 & 0.359 \\
\hline
\end{tabular}

N-114, normal control tissues labeled with 114 iTRAQ tag; D-116, dysplasia tissues labeled with 116 iTRAQ tag; C-119, carcinoma tissues labeled with 119 iTRAQ tag.

clinicopathological characteristics of the patients are shown in Table II. Moreover, similar characteristics were observed in the animal model. As shown in Fig. 1D, Trx-1 expression was increased in the dysplasia tissues $(5.4 \pm 0.33)$ compared with the control tissues $(3.93 \pm 0.39 ; \mathrm{P}<0.05)$, as well as the tongue carcinogenesis tissues $(5.91 \pm 0.16)$, depending on the extent and intensity of staining.

Inhibition of Trx-1 suppresses the proliferation and invasion, and enhances the apoptosis of OSCC cells under hypoxic conditions. In solid tumors, hypoxia is a common feature that leads to oxidative stress and results in redox imbalance (19). It has been demonstrated that hypoxia is involved in the growth and aggressiveness of many types of cancer (20-22). In this study, two OSCC cell lines, CAL33 and HSC6, were cultured under hypoxic conditions for 12, 24 and $48 \mathrm{~h}$. Cell proliferation was significantly increased compared with normoxic conditions, as demonstrated by CCK-8 assays $(\mathrm{P}<0.05)$ (Fig. $2 \mathrm{~A}$ and $\mathrm{B})$. The cell invasive ability was also promoted at $24 \mathrm{~h}$, as demonstrated by Transwell assay $(\mathrm{P}<0.05)$ (Fig. 2C). Moreover, Trx-1 expression was detected by western blot analysis. The cells were cultured under hypoxic conditions for $3,6,12$ and $24 \mathrm{~h}$. Trx-1 expression gradually increased in a time-dependent manner upon hypoxic stimulation, as was the expression of HIF-1 $\alpha(\mathrm{P}<0.05)$ (Fig. 2D and $\mathrm{E})$.
Subsequently, experiments were carried out to examine the effect of Trx-1 on the phenotypes of OSCC cells under hypoxic conditions. When Trx-1 was specifically inhibited by PX-12 at various concentrations (data not shown), cell viability and the cell invasive capacity gradually decreased in a dose-dependent manner $(\mathrm{P}<0.05)$ (Fig. 3A and B). Moreover, the apoptosis of the OSCC cells was measured by flow cytometry. As a result, the percentages of OSCC cells undergoing early apoptosis were increased by treatment with $\mathrm{PX}-12$ in a concentrationdependent manner $(\mathrm{P}<0.05)$ (Fig. 3C).

Inhibition of Trx-1 induces the apoptosis of OSCC cells in a ROS-dependent manner under hypoxic conditions. Since the inhibition of the activation of Trx-1 has been hypothesized to affect the redox state of cells (23), the intracellular ROS levels of OSCC cells were detected in the presence of PX-12 $(10,20$ or $4 \mu \mathrm{M})$ for $24 \mathrm{~h}$, and were markedly increased compared with those of controls under hypoxic conditions $(\mathrm{P}<0.05)$ (Fig. 4A). Treatment of the OSCC cells with the antioxidant agent, NAC (5 mM), $1 \mathrm{~h}$ prior to the addition of PX-12 caused a significant decrease in ROS levels $(\mathrm{P}<0.05)$ (Fig. 4B and $\mathrm{C}$ ) and markedly prevented cellular apoptosis $(\mathrm{P}<0.05)$ (Fig. 4D and E). Furthermore, the addition of NAC also attenuated the inhibitory effects of PX-12 on cell proliferation and invasion (data not shown). These results demonstrated that the effects of PX-12 were ROS-dependent. 
Table II. The clinicopathological characteristics of all the patients.

\begin{tabular}{|c|c|c|c|}
\hline Characteristics & $\begin{array}{l}\text { Normal oral } \\
\text { mucosa } \\
(n=10)\end{array}$ & $\begin{array}{c}\text { Oral } \\
\text { leukoplakia } \\
(n=15)\end{array}$ & $\begin{array}{l}\text { OSCC } \\
(n=35)\end{array}$ \\
\hline Age (years) & $37.8 \pm 13.96$ & $52.13 \pm 11.60$ & $55.97 \pm 10.29$ \\
\hline \multicolumn{4}{|l|}{ Sex } \\
\hline Male & 4 & 11 & 25 \\
\hline Female & 6 & 4 & 10 \\
\hline \multicolumn{4}{|l|}{ Smoking history } \\
\hline Yes & 3 & 10 & 18 \\
\hline No & 7 & 5 & 17 \\
\hline \multicolumn{4}{|l|}{$\begin{array}{l}\text { Alcohol } \\
\text { comsumption }\end{array}$} \\
\hline Yes & 2 & 4 & 9 \\
\hline No & 8 & 11 & 26 \\
\hline \multicolumn{4}{|l|}{ Site } \\
\hline Tongue & - & 12 & 24 \\
\hline Bucca cavioris & 6 & 3 & 2 \\
\hline Gingiva & 4 & - & 6 \\
\hline Palate & - & - & 3 \\
\hline \multicolumn{4}{|l|}{$\begin{array}{l}\text { Histological grade } \\
\text { of dysplasia }\end{array}$} \\
\hline Mild & - & 2 & - \\
\hline Moderate & - & 5 & - \\
\hline Severe & - & 8 & - \\
\hline \multicolumn{4}{|l|}{$\begin{array}{l}\text { Histological grade } \\
\text { of tumor }\end{array}$} \\
\hline Well & - & - & 14 \\
\hline Moderate & - & - & 10 \\
\hline Poor & - & - & 11 \\
\hline \multicolumn{4}{|l|}{ TNM stage } \\
\hline $\mathrm{I}$ & - & - & 11 \\
\hline II & - & - & 13 \\
\hline III & - & - & 5 \\
\hline IV & - & - & 6 \\
\hline
\end{tabular}

In addition, the mechanisms underlying the apoptosispromoting effects of PX-12 in OSCC cells were assessed by western blot analysis. The levels of apoptosis-related proteins, such as the anti-apoptotic protein, Bcl-2, the pro-apoptotic protein, Bax, and PARP cleavage (Cle-PARP) were examined in the total protein from CAL33 and HSC6 cells. As shown in Fig. 5, the expression of Bax and Cle-PARP was upregulated and the expression of $\mathrm{Bcl}-2$ was downregulated by $\mathrm{PX}-12$ under hypoxic conditions. Additionally, the ratio of Bcl-2/Bax was decreased. However, pretreatment with NAC reversed the changes in Bax, Bcl-2 and Cle-PARP expression, and in the ratio of Bcl-2/Bax. Thus, these results suggested that the inhibition of Trx-1 induced apoptosis via ROS accumulation.

Inhibition of Trx-1 delays $4 N Q O$-induced oral carcinogenesis in vivo. The in vitro study results indicated that Trx-1 was a promising therapeutic target for oral carcinogenesis. Thus, in our in vivo experiments, we randomly subdivided the rats exposed to 4NQO into the PX-12 treatment group $(n=8)$ and the disease-control group $(n=8)$ at week 18 , when dysplasia was clearly observed. The rats were administered PX-12 $(12 \mathrm{mg} / \mathrm{kg})$ via tail vein injection weekly, from week 18 to the end of the experiment (Fig. 6A). The gross weight and emergence rate of the tumor was measured periodically. Notably, the PX-12-treated rats had a lower cancerization rate (3/8) than the rats in the disease-control group (7/8) (Fig. 6B and C). In addition, the weights of the rats in the PX-12 treatment group $(417.63 \pm 13.22 \mathrm{~g})$ were higher than those of the rats in the disease-control group $(356.38 \pm 19.56 \mathrm{~g})(\mathrm{P}<0.05)$ (Fig. 6D). Additionally, apoptosis was determined by TUNEL assay and the expression of Bax was detected by IHC. As shown in Fig. 6E, the apoptotic rate of the PX-12 treatment group appeared higher than that of the disease-control group $(9.93 \pm 1.86$ vs. $2.09 \pm 0.61 \%, \mathrm{P}<0.05)$. Similarly, the expression of Bax was increased in the PX-12 treatment group $(5.6 \pm 0.4, \mathrm{P}<0.05)$. These results demonstrated that Trx-1 may be a preventative and therapeutic target during oral epithelial malignant transformation.

\section{Discussion}

Oral carcinogenesis is a complex and multifaceted process, and the majority of cases begin with epithelial dysplasia lesions $(3,4)$. However, effective interference efforts to end or delay oral leukoplakia or erythroplakia from undergoing malignant transformation remain limited, which may be due to the complex causes, lengthy course and individual differences. With the use of a mature animal model, the rat model of 4NQO-induced oral carcinogenesis, studies are able to include the whole process of the disease while reducing the individual differences. In the present study, iTRAQ-labeled quantitative proteomics analysis was performed to disclose the molecular alterations at different stages during 4NQO-induced oral carcinogenesis.

In this study, the high-throughput results demonstrated that the expression of oxidative stress-associated proteins was altered significantly, which was consistent with the experimental results on solid tumors. Several systematic reviews and meta-analyses have reported that tobacco use and alcohol consumption are risk factors for oral cancer (24). The characteristic increased levels of oxidative stress in cancer cells result from an imbalance between the generation and elimination of ROS (25). It has been reported that oxidative stress contributes to oral epithelial malignant progression from dysplasia to carcinogenesis (4). As a common feature of solid tumors, hypoxia causes oxidative stress and results in a redox imbalance in the tumor microenvironment (26). Some researchers have reported that the dysfunction and deregulation of hypoxia-related proteins are often an early event during oral carcinogenesis $(27,28)$.

Human Trx-1 belongs to a family of small redox proteins that are reduced by thioredoxin reductase and NADPH, following the reduction of oxidative target proteins (29). Trx-1 plays an important role in maintaining the redox balance, which is essential for cell survival, tumor development and angiogenesis (30). It has been found to be upregulated in 
A

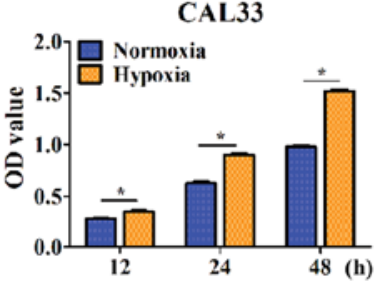

C

B
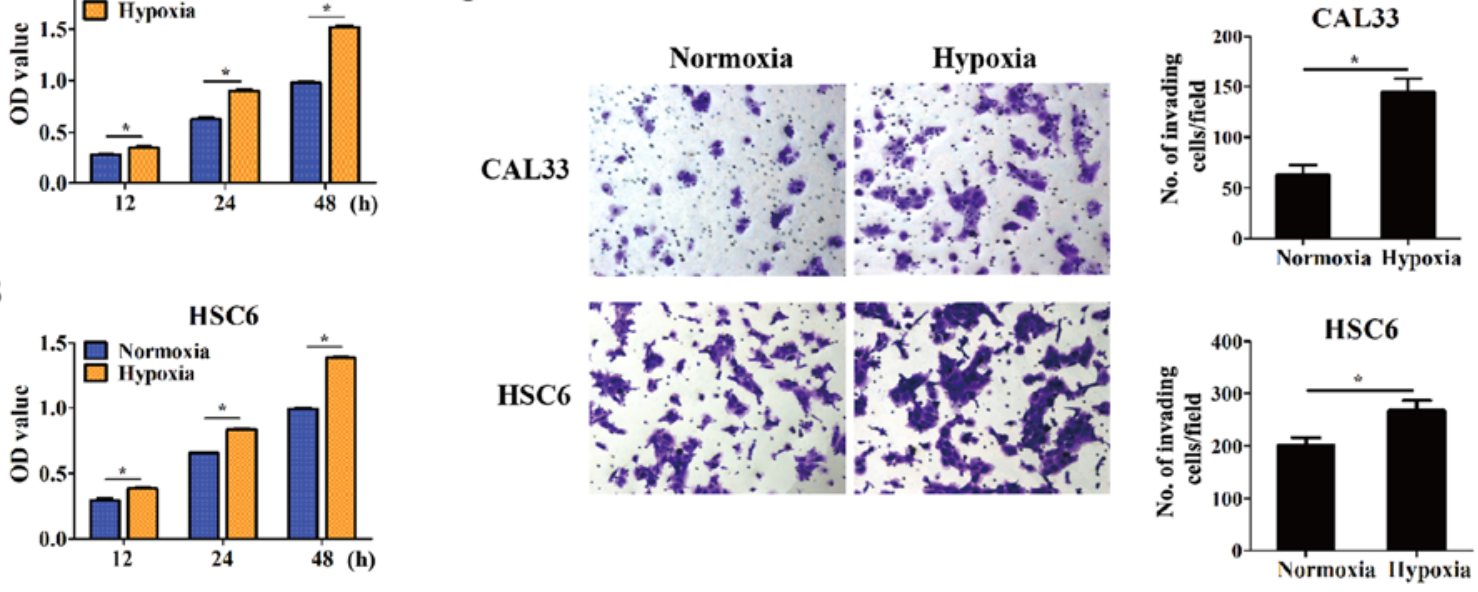

D

$\mathbf{E}$
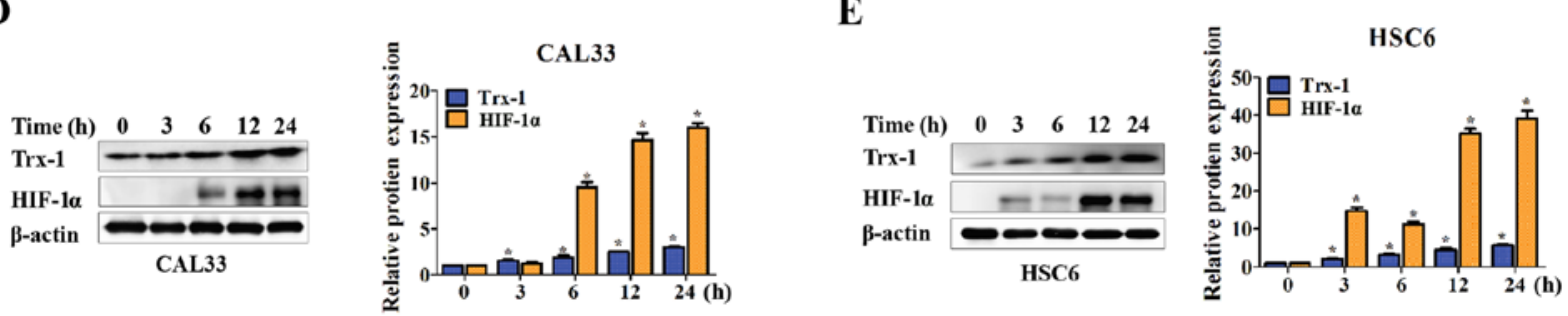

Figure 2. Hypoxia renders oral squamous cell carcinoma (OSCC) cells capable of proliferation and invasion. (A and B) Cell viability was detected by cell counting kit-8 (CCK-8) assays after culturing the CAL33 and HSC6 cells for 12, 24 and $48 \mathrm{~h}$ under hypoxic conditions (OD values at $450 \mathrm{~nm}$ are shown as the means \pm standard deviation from 3 independent experiments); ${ }^{*} \mathrm{P}<0.05$ vs. normoxic conditions. (C) Transwell assays were performed to determine the invasive ability of CAL33 and HSC6 cells. Cells were allowed to invade for $24 \mathrm{~h}$ under hypoxic or normoxic conditions. Invading cell numbers were counted in 5 random fields and are shown as the means \pm standard deviation from 3 independent experiments (magnification, $x 200$ ); "P<0.05. (D and E) CAL33 and HSC6 cells were cultured under hypoxic conditions for 0,3,6,12 and $24 \mathrm{~h}$. Western blot analysis was used to determine the expression of thioredoxin-1 (Trx-1) and HIF-1 $\alpha$ (relative ratio of band intensity was normalized to the control); ${ }^{*} \mathrm{P}<0.05$ vs. control.

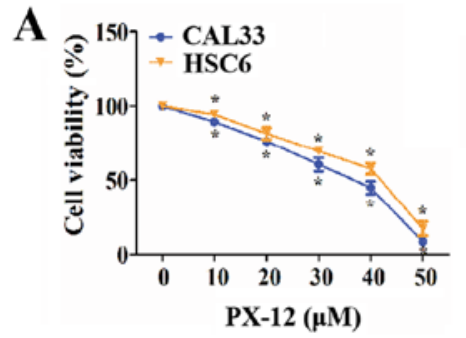

B

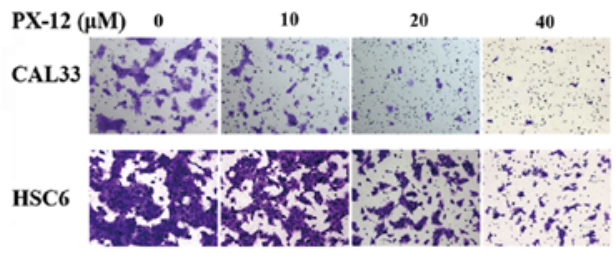

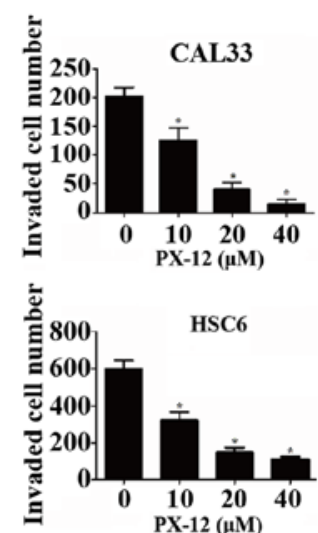

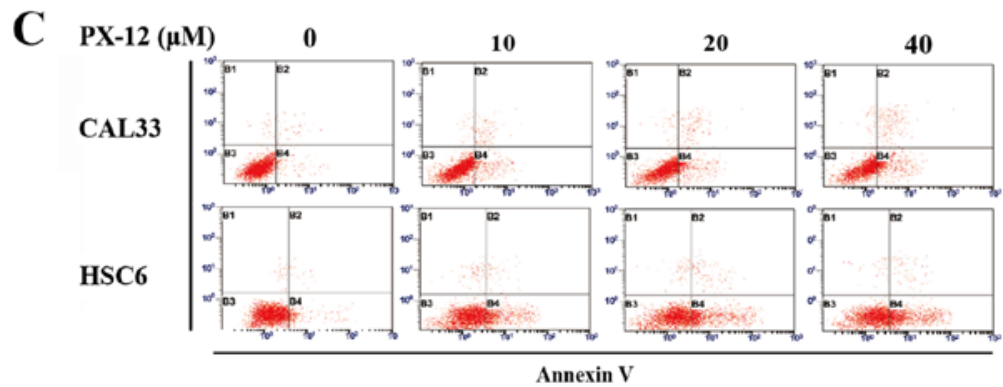

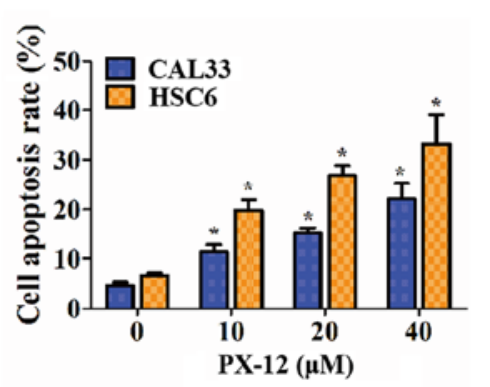

Figure 3. Inhibition of thioredoxin-1 (Trx-1) suppresses the proliferation and invasion, and enhances the apoptosis of oral squamous cell carcinoma (OSCC) cells under hypoxic conditions. The CAL33 and HSC6 cells were incubated for $24 \mathrm{~h}$ with increasing concentrations of PX-12 (0-50 $\mu \mathrm{M})$ under hypoxic conditions. Cell counting kit-8 (CCK-8) and Transwell assays were performed to test the (A) cell viability and (B) invasive ability of the CAL33 and HSC6 cells, after specifically inhibiting Trx-1 with PX-12. Cell viability was normalized to the control. Data are shown as the means \pm standard deviation for three independent experiments; ${ }^{\mathrm{P}}<0.05$ vs. control. (C) Annexin V/PI staining was used to examine cellular apoptosis. The percentages of OSCC cells undergoing early apoptosis are shown as the means \pm standard deviation from 3 independent experiments; " $\mathrm{P}<0.05$ vs. control. 

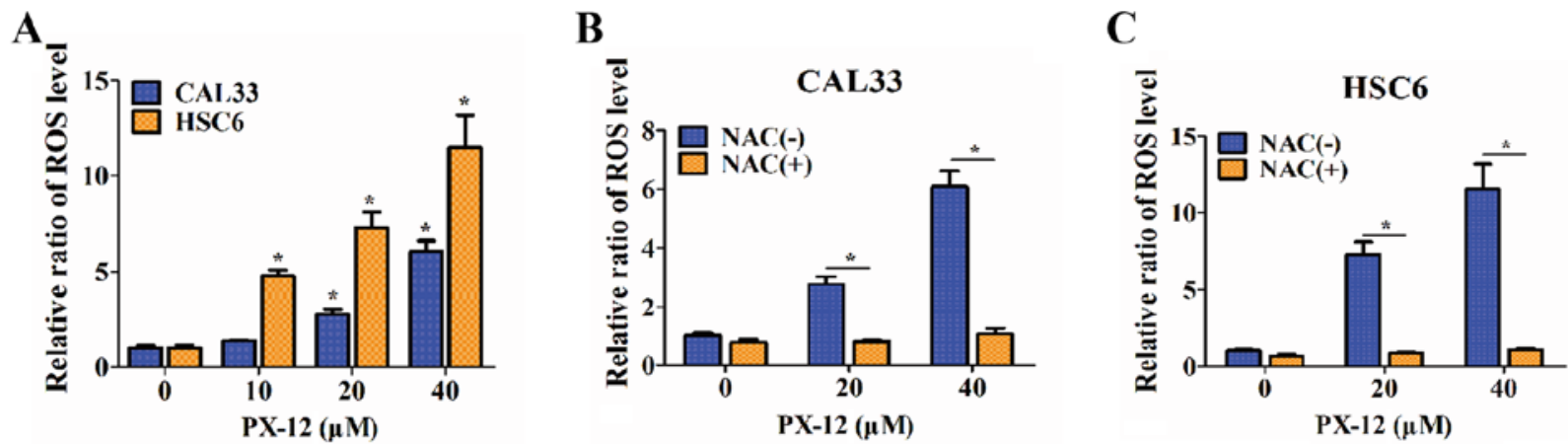

D

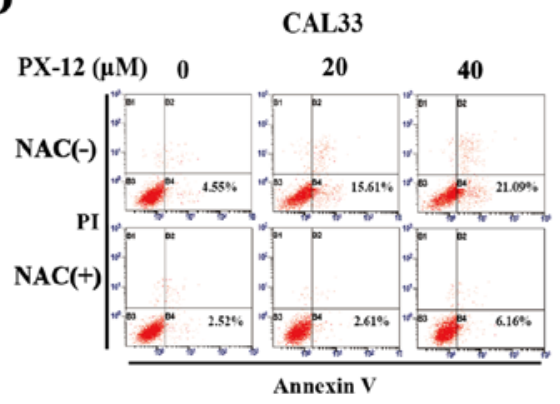

$\mathbf{E}$

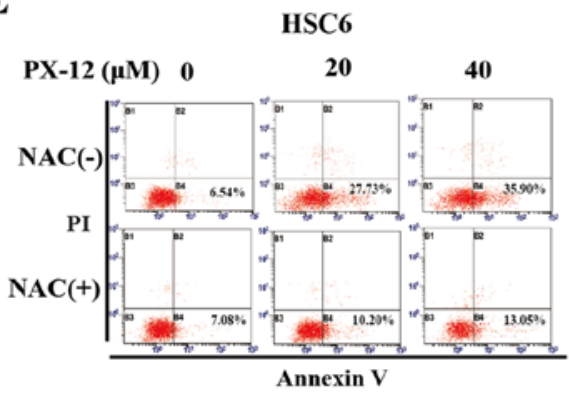

Figure 4. Inhibition of thioredoxin-1 (Trx-1) induces the apoptosis of oral squamous cell carcinoma (OSCC) cells in a reactive oxygen species (ROS)-dependent manner under hypoxic conditions. The CAL33 and HSC6 cells were incubated with PX-12 (0, 10, 20 or $40 \mu \mathrm{M})$ for $24 \mathrm{~h}$. (A) 2,7-Dichlorodihydrofluorescein diacetate (DCFH-DA) was used to detect the ROS levels (relative ratio of mean fluorescence intensity was normalized to the control); ${ }^{\circ} \mathrm{P}<0.05$. (B and C) The CAL33 and HSC6 cells were treated with NAC $(5 \mathrm{mM})$ at $1 \mathrm{~h}$ prior to the addition of PX-12 $(0,20$ or $40 \mu \mathrm{M})$ for a further $24 \mathrm{~h}$. DCFH-DA was used to measure the ROS levels; ${ }^{*} \mathrm{P}<0.05$. (D and E) Annexin V/PI staining was used to examine cellular apoptosis; ${ }^{*} \mathrm{P}<0.05$.

A
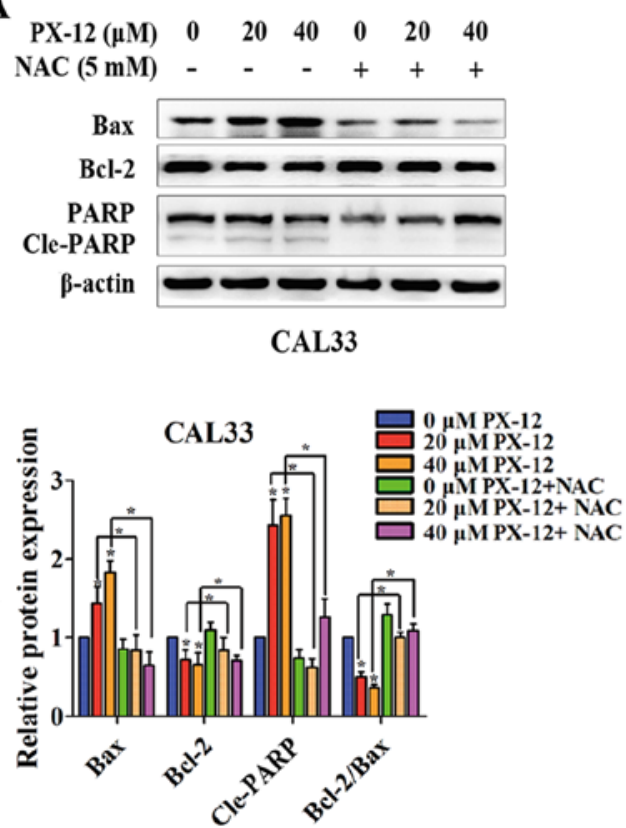

B

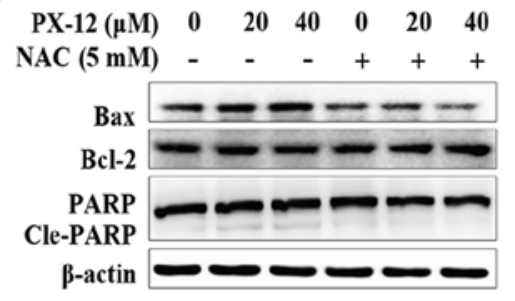

HSC6

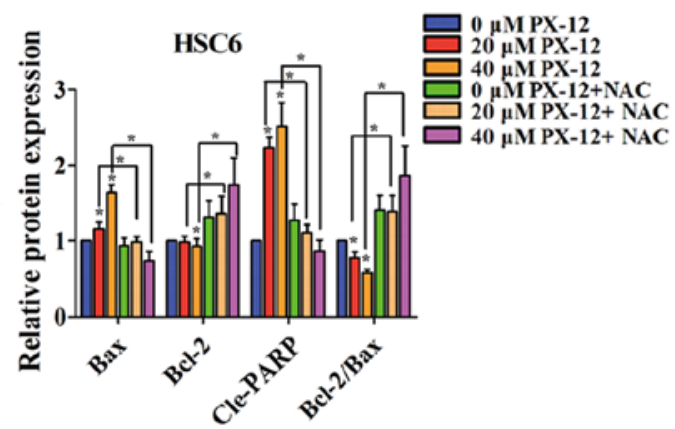

Figure 5. Underlying mechanisms of the apoptosis-inducing effect of PX-12 on oral squamous cell carcinoma (OSCC) cells. (A and B) The CAL33 and HSC6 cells were treated with or without NAC $(5 \mathrm{mM})$ at $1 \mathrm{~h}$ prior to the addition of PX-12 $(0,20$ or $40 \mu \mathrm{M})$ for a further $24 \mathrm{~h}$. Western blot analysis was performed to detect the expression of the anti-apoptotic protein, Bcl-2, and the pro-apoptotic proteins, Bax and cleaved (Cle-)PARP (relative ratio of band intensity was normalized to the control); ${ }^{*} \mathrm{P}<0.05$.

many types of cancer $(23,31-33)$ and is regarded as a target for cancer therapy $(34,35)$. In this study, we investigated the role of Trx-1 in the development of oxidative stress-associated oral epithelial malignancy. The results revealed that the specific inhibition of Trx-1 induced apoptosis under hypoxic conditions via ROS accumulation in OSCC cells, which was consistent with results in other solid tumors (36). The role of ROS in tumorigenesis has been controversial for several years (37). It 
$\mathbf{A}_{0}$

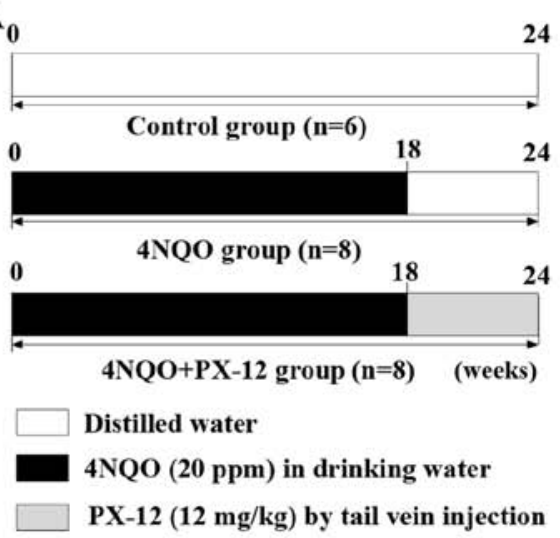

B

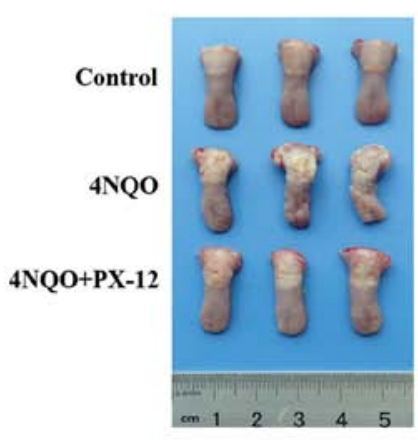

C

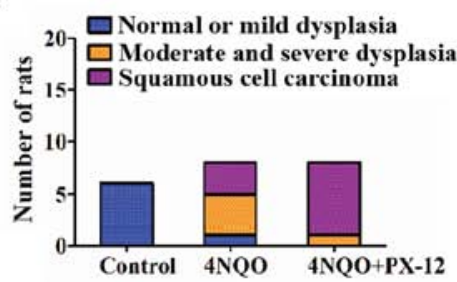

D

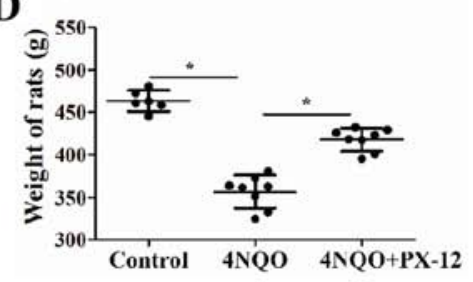

$\mathbf{E}$
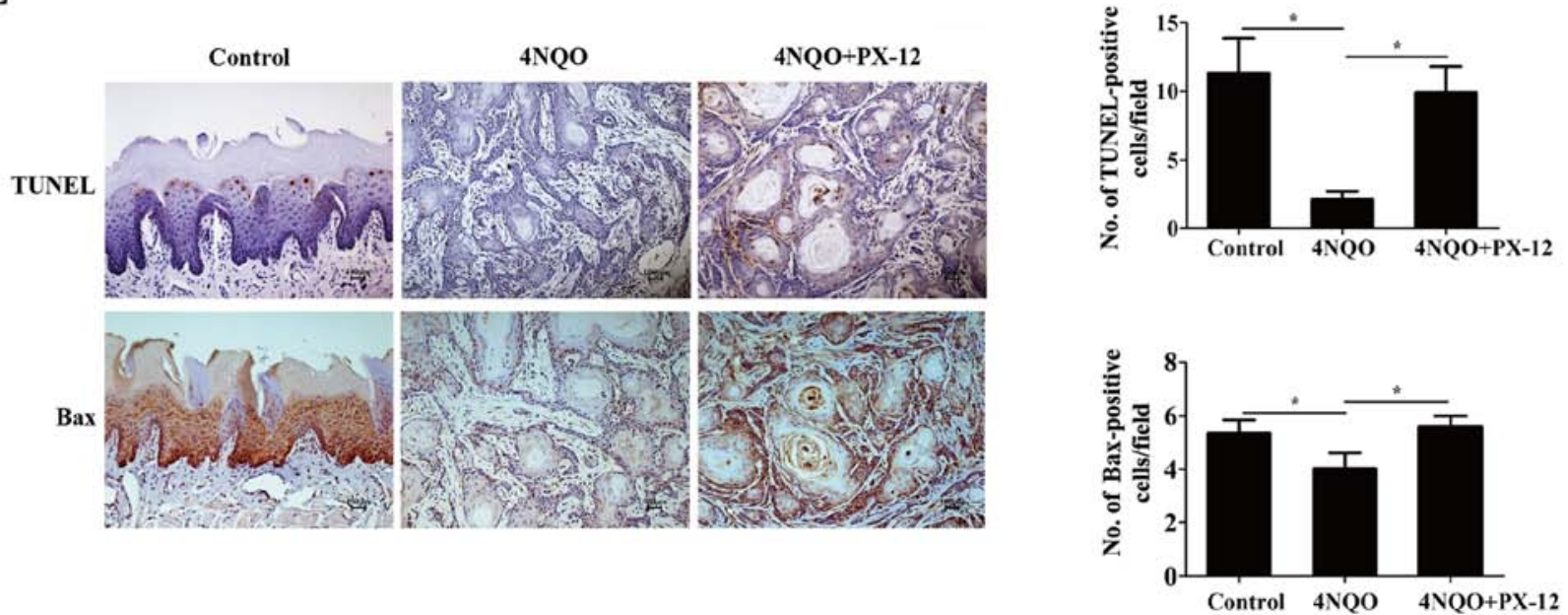

Figure 6. Inhibition of thioredoxin-1 (Trx-1) delays 4-nitroquinoline 1-oxide (4NQO)-induced oral carcinogenesis in vivo. (A) Schematic diagram of PX-12 intervention during 4NQO-induced rat oral carcinogenesis. (B) Representative tissue samples are shown. (C) Histological distribution and (D) weights among the 3 groups. (E) TUNEL assay was performed to examine cell apoptosis in the control, 4NQO and PX-12 intervention groups. Positive cell rates are shown as the means \pm standard deviation. The expression of Bax was measured by immunohistochemistry (IHC) staining. IHC scores are shown as the means \pm standard deviation. Magnification, $\mathrm{x} 200 ;{ }^{*} \mathrm{P}<0.05$.

may play a dual role in cell survival. In a previous study, it was found that interleukin (IL)-1 $\beta$ promoted the invasive ability of OSCC CAL27 cells by upregulating ROS (38). In the present study, it was suggested that the excessive accumulation of intracellular ROS through the specific inhibition of Trx-1 was due to the apoptosis of the OSCC cell lines, CAL33 and HSC6. These results suggest that Trx-1 may be a therapeutic target for OSCC prevention and therapy.

To further validate the hypothesis, the preventive effect of Trx-1 inhibition was tested with a rat model of 4NQO-induced oral carcinogenesis. A specific inhibitor of Trx-1 (PX-12) was administered via tail vein injection when dysplasia lesions had developed. In general, by comparing the gross weight and cancerization rate, the rats treated with PX-12 were in a better condition than the disease controls when exposed to the same carcinogen. No obvious injury to the liver or kidneys was observed in the PX-12 treatment group (data not shown). The results suggested that the inhibition of Trx-1 may provide a promising chemoprevention strategy with which to interrupt oral malignant transformation. Although the antitumor effects of PX-12 have been previously reported $(15,36)$, the present study investigated the preventative effect of $\mathrm{PX}-12$ in potentially malignant disorders by continual observation of the $4 \mathrm{NQO}$ rat model in vivo. These results are hopefully more objective than those from a xenograft model or in vitro studies (15).

In conclusion, this was a preliminary study examining the oxidative stress-associated proteins during oral malignant transformation in vivo and in vitro. In this study, it was demonstrated that the inhibition of Trx-1 in OPMDs may be a potential target for delaying hypoxia-induced oral malignant transformation, and that this chemopreventive effect is mediated in a ROS-dependent manner. These results open up the possibility for prevention and early intervention strategies for OSCC, and are worthy of further research in the future.

\section{Acknowledgements}

This study was supported by the Science and Technology Planning Project of Guangdong Province, China (grant nos. 2014A020212104 and 2014A020212081). 


\section{Competing interests}

The authors declare that they have no competing interests.

\section{References}

1. Chen W, Zheng R, Baade PD, Zhang S, Zeng H, Bray F, Jemal A, Yu XQ and He J: Cancer statistics in China, 2015. CA Cancer J Clin 66: 115-132, 2016

2. Siegel RL, Miller KD and Jemal A: Cancer statistics, 2015. CA Cancer J Clin 65: 5-29, 2015.

3. Arduino PG, Bagan J, El-Naggar AK and Carrozzo M: Urban legends series: Oral leukoplakia. Oral Dis 19: 642-659, 2013.

4. Choudhari SK, Chaudhary M, Gadbail AR, Sharma A and Tekade S: Oxidative and antioxidative mechanisms in oral cancer and precancer: A review. Oral Oncol 50: 10-18, 2014.

5. Zhang X, Han S, Han HY, Ryu MH, Kim KY, Choi EJ, Cha IH and Kim J: Risk prediction for malignant conversion of oral epithelial dysplasia by hypoxia related protein expression. Pathology 45: 478-483, 2013.

6. Dionne KR, Warnakulasuriya S, Zain RB and Cheong SC: Potentially malignant disorders of the oral cavity: Current practice and future directions in the clinic and laboratory. Int J Cancer 136: 503-515, 2015.

7. Liu F, Zhang Y, Men T, Jiang X, Yang C, Li H, Wei X, Yan D, Feng G, Yang J, et al: Quantitative proteomic analysis of gastric cancer tissue reveals novel proteins in platelet-derived growth factor b signaling pathway. Oncotarget 8: 22059-22075, 2017.

8. Wang Z, Jiang L, Huang C, Li Z, Chen L, Gou L, Chen P, Tong A, Tang M, Gao F, et al: Comparative proteomics approach to screening of potential diagnostic and therapeutic targets for oral squamous cell carcinoma. Mol Cell Proteomics 7: 1639-1650, 2008.

9. Qing S, Tulake W, Ru M, Li X, Yuemaier R, Lidifu D, Rouzibilali A, Hasimu A, Yang Y, Rouziahong R, et al: Proteomic identification of potential biomarkers for cervical squamous cell carcinoma and human papillomavirus infection. Tumour Biol 39: $1010428317697547,2017$.

10. Dey KK, Pal I, Bharti R, Dey G, Kumar BN, Rajput S, Parekh A, Parida S, Halder P, Kulavi I, et al: Identification of RAB2A and PRDX1 as the potential biomarkers for oral squamous cell carcinoma using mass spectrometry-based comparative proteomic approach. Tumour Biol 36: 9829-9837, 2015.

11. Hung KF, Liu CJ, Chiu PC, Lin JS, Chang KW, Shih WY, Kao SY and Tu HF: MicroRNA-31 upregulation predicts increased risk of progression of oral potentially malignant disorder. Oral Oncol 53: 42-47, 2016

12. Kanojia D and Vaidya MM: 4-nitroquinoline-1-oxide induced experimental oral carcinogenesis. Oral Oncol 42: 655-667, 2006

13. Wu T, Hong Y, Jia L, Wu J, Xia J, Wang J, Hu Q and Cheng B: Modulation of IL-1 $\beta$ reprogrammes the tumor microenvironment to interrupt oral carcinogenesis. Sci Rep 6: 20208, 2016.

14. Lan A, Li W, Liu Y, Xiong Z, Zhang X, Zhou S, Palko O, Chen H, Kapita M, Prigge JR, et al: Chemoprevention of oxidative stressassociated oral carcinogenesis by sulforaphane depends on NRF2 and the isothiocyanate moiety. Oncotarget 7: 53502-53514, 2016.

15. Li GZ, Liang HF, Liao B, Zhang L, Ni YA, Zhou HH, Zhang EL, Zhang BX and Chen XP: PX-12 inhibits the growth of hepatocelluar carcinoma by inducing S-phase arrest, ROS-dependent apoptosis and enhances 5-FU cytotoxicity. Am J Transl Res 7: $1528-1540,2015$.

16. Hong Y, Yang L, Li C, Xia H, Rhodus NL and Cheng B: Frequent mutation of $\mathrm{p} 16(\mathrm{CDKN} 2 \mathrm{~A})$ exon 1 during rat tongue carcinogenesis induced by 4-nitroquinoline-1-oxide. Mol Carcinog 46: 85-90, 2007.

17. Jiang X, Wang J, Chen X, Hong Y, Wu T, Chen X, Xia J and Cheng B: Elevated autocrine chemokine ligand 18 expression promotes oral cancer cell growth and invasion via Akt activation. Oncotarget 7: 16262-16272, 2016.

18. Hanschmann EM, Godoy JR, Berndt C, Hudemann C and Lillig $\mathrm{CH}$ : Thioredoxins, glutaredoxins, and peroxiredoxins molecular mechanisms and health significance: From cofactors to antioxidants to redox signaling. Antioxid Redox Signal 19: $1539-1605,2013$
19. Höckel M and Vaupel P: Tumor hypoxia: Definitions and current clinical, biologic, and molecular aspects. J Natl Cancer Inst 93: 266-276, 2001 .

20. Zhang L, Hu Y, Xi N, Song J, Huang W, Song S, Liu Y, Liu X and Xie Y: Partial oxygen pressure affects the expression of prognostic biomarkers HIF-1 alpha, Ki67, and CK20 in the microenvironment of colorectal cancer tissue. Oxid Med Cell Longev 2016: 1204715, 2016.

21. Zheng Y, Ni Y, Huang X, Wang Z and Han W: Overexpression of HIF-1 $\alpha$ indicates a poor prognosis in tongue carcinoma and may be associated with tumour metastasis. Oncol Lett 5: 1285-1289, 2013.

22. Liu Z, Tu K, Wang Y, Yao B, Li Q, Wang L, Dou C, Liu Q and Zheng X: Hypoxia accelerates aggressiveness of hepatocellular carcinoma cells involving oxidative stress, epithelial-mesenchymal transition and non-canonical hedgehog signaling. Cell Physiol Biochem 44: 1856-1868, 2017.

23. Noike T, Miwa S, Soeda J, Kobayashi A and Miyagawa S: Increased expression of thioredoxin-1, vascular endothelial growth factor, and redox factor-1 is associated with poor prognosis in patients with liver metastasis from colorectal cancer. Hum Pathol 39: 201-208, 2008.

24. Mishra R: Glycogen synthase kinase 3 beta: Can it be a target for oral cancer. Mol Cancer 9: 144, 2010.

25. Cairns RA, Harris IS and Mak TW: Regulation of cancer cell metabolism. Nat Rev Cancer 11: 85-95, 2011.

26. Zhou J, Schmid T, Schnitzer S and Brüne B: Tumor hypoxia and cancer progression. Cancer Lett 237: 10-21, 2006.

27. DE Lima PO, Jorge CC, Oliveira DT and Pereira MC: Hypoxic condition and prognosis in oral squamous cell carcinoma. Anticancer Res 34: 605-612, 2014.

28. Kujan O, Shearston K and Farah CS: The role of hypoxia in oral cancer and potentially malignant disorders: A review. J Oral Pathol Med 46: 246-252, 2017.

29. Sahaf B, Söderberg A, Spyrou G, Barral AM, Pekkari K, Holmgren A and Rosén A: Thioredoxin expression and localization in human cell lines: Detection of full-length and truncated species. Exp Cell Res 236: 181-192, 1997.

30. Kakolyris S, Giatromanolaki A, Koukourakis M, Powis G, Souglakos J, Sivridis E, Georgoulias V, Gatter KC and Harris AL: Thioredoxin expression is associated with lymph node status and prognosis in early operable non-small cell lung cancer. Clin Cancer Res 7: 3087-3091, 2001.

31. Zhu X, Huang C and Peng B: Overexpression of thioredoxin system proteins predicts poor prognosis in patients with squamous cell carcinoma of the tongue. Oral Oncol 47: 609-614, 2011.

32. Li C, Thompson MA, Tamayo AT, Zuo Z, Lee J, Vega F, Ford RJ and Pham LV: Over-expression of Thioredoxin-1 mediates growth, survival, and chemoresistance and is a druggable target in diffuse large B-cell lymphoma. Oncotarget 3: 314-326, 2012.

33. Bhatia M, McGrath KL, Di Trapani G, Charoentong P, Shah F, King MM, Clarke FM and Tonissen KF: The thioredoxin system in breast cancer cell invasion and migration. Redox Biol 8: 68-78, 2016.

34. Powis G and Kirkpatrick DL: Thioredoxin signaling as a target for cancer therapy. Curr Opin Pharmacol 7: 392-397, 2007.

35. Roh JL, Jang H, Kim EH and Shin D: Targeting of the glutathione, thioredoxin, and Nrf2 antioxidant systems in head and neck cancer. Antioxid Redox Signal 27: 106-114, 2017.

36. You BR, Shin HR, Han BR and Park WH: PX-12 induces apoptosis in Calu-6 cells in an oxidative stress-dependent manner. Tumour Biol 36: 2087-2095, 2015.

37. Di Meo S, Reed TT, Venditti P and Victor VM: Harmful and beneficial role of ROS. Oxid Med Cell Longev 2016: 7909186, 2016.

38. Chen X,Lv Q, Hong Y, Chen X, Cheng B and Wu T: IL-1 $\beta$ maintains the redox balance by regulating glutaredoxin 1 expression during oral carcinogenesis. J Oral Pathol Med 46: 332-339, 2017.

This work is licensed under a Creative Commons Attribution-NonCommercial-NoDerivatives 4.0 International (CC BY-NC-ND 4.0) License. 